\title{
Pregnancy and Covid-19: Some Thing to Worry About?
}

\author{
Samreen Iqbal, Muhammad Majid Ali Jafri
}

How to cite this Article:

Iqbal S, Jafri MMA. Pregnancy and Covid-19: Some Thing to Worry About? J Bahria Uni Med Dental Coll. 2020;10(3): 169-70 DOI: https://doi.org/10.51985/JBUMDC2020044

Pregnancy is a special time, full of excitement and anticipation, but for expectant mothers facing the outbreak of the coronavirus disease (COVID-19), fear, anxiety and uncertainty are clouding this otherwise happy time.

Coronavirus disease 2019 (COVID-19) has led to the deadliest pandemic observed in more than a 100 years. ${ }^{1}$ Around 131 million women give birth annually. This population is particularly vulnerable to emerging infectious pathogens due to alterations in immune, respiratory, and cardiovascular physiology that occurs during pregnancy. Recent outbreaks of severe acute respiratory syndrome, Middle East respiratory syndrome, influenza H1N1, Ebola virus disease, and Zika virus disease resulted in high rates of maternal morbidity and mortality, fetal loss, and fetal harm. Fortunately early data regarding pregnancy outcomes in COVID-19 are reassuring: maternal outcomes are similar to non-pregnant adults; vertical transmission and neonatal infection are rare. However pregnant women remain at risk of severe disease requiring intensive care, and they deserve equity to access therapeutic options guided by rigorous scientific data. ${ }^{2-3}$ More than $90 \%$ of infected mothers recovered without undergoing delivery. ${ }^{3}$

Pregnant women should follow the same recommendations as non pregnant individuals to avoid exposure to the virus such as social distancing, hand hygiene, disinfecting surfaces and wearing a mask in public. All pregnant women should be monitored for development of symptoms and signs of COVID-19, particularly if they have had close contact with a confirmed case or persons under investigation.

American College of Obstetricians and Gynecologists (ACOG) has developed algorithms to evaluate pregnant outpatients with suspected or confirmed COVID-194.Patients having symptoms compatible with COVID-19, the severity of illness, underlying co-morbidities, and clinical status should be assessed to determine the potential need for hospitalization.

Possible vertical transmission has been reported in several cases of peripartum maternal infection in the third trimester,

$\begin{array}{ll}\text { Samreen Iqbal } & \text { | } \\ \text { Assistant Professor, Department of Obsrtrics and Gynaecology, } \\ \text { Eahria University Medical \& Dental College, Karachi } \\ \text { Muhail: drsam222@ yahoo.com } \\ \text { Peads Specialist } \\ \text { Agha Khan University Hospital, Karachi } \\ \text { Received: 22-Apr-2020 }\end{array}$

suggesting congenital infection is possible but uncommon. ${ }^{3}$ The placentas from COVID-19- positive pregnant women showed injury; type of injury seen in the placentas revealed abnormal blood flow between the mothers and their babies in utero, pointing to a new complication of COVID-19. An early diagnosis could help in persistent clinical monitoring of pregnant women during the pandemic. ${ }^{5}$ Complications of infection include acute respiratory distress syndrome, arrhythmias, acute cardiac injury, shock, preterm birth, fetal growth retardation and perinatal mortality. ${ }^{6}$ In most cases, the timing of delivery should be dictated by obstetric indications rather than maternal diagnosis of COVID-19. There is no alteration in usual timings of delivery indicated among women with suspected or confirmed COVID-19 infection early in pregnancy who then recovered.

For women with suspected or confirmed COVID-19 in the third trimester, it is advisable to postpone delivery (if no other medical indications arise) until a negative test result is obtained or quarantine restrictions are lifted to avoid virus transmission to the neonate..$^{8-11}$

In general, a diagnosis of COVID-19 during pregnancy is not an indication for early delivery. ${ }^{12}$ Neither is COVID-19 an indication to alter the route of delivery. Cesarean delivery is performed for standard obstetric indications, which may include acute decompensation of mother with COVID-19. ${ }^{7}$ Corona virus has not been found in breast milk, however if the mother has COVID-19 infection, she can spread the virus to the infant through tiny droplets that spread when she talks, coughs or sneezes. ${ }^{7-12}$

Congenital infection in a live born infant depends on presence or absence of clinical features of infection in a newborn and mother with COVID-19 infection. In symptomatic cases, congenital infection is confirmed if the virus is detected by PCR in umbilical cord blood or neonatal blood collected within the first 12 hours of birth or amniotic fluid collected prior to rupture of membranes. In asymptomatic cases, neonatal infection is confirmed if the virus is detected by PCR in cord blood or neonatal blood collected within 12 hours of birth. Neonatal infection may be acquired intrapartum or acquired postpartum. ${ }^{13,14}$

The multidisciplinary team in contact with the newborn must wear PPE (N95 or PFF2 masks, glasses and face protectors, waterproof aprons, and sterile gloves and caps). Newborns requiring intensive care should ideally be admitted to an isolation room with the potential for negative ambient pressure or other available air filtration systems. Incubators should be kept at a minimum distance of 1.5 meters. For 
newborns with indications for non-invasive ventilation (CPAP or bilevel), high-flow oxygen therapy, or invasive mechanical ventilation, precautions should be taken to avoid aerosols, droplets, and contact through the use of PPEs recommended for this purpose ${ }^{13,14} \mathrm{It}$ is satisfactory that most newborn babies infected with the virus recovered uneventfully. Unfortunately, pregnant women have systematically been excluded from clinical trials of therapeutics and vaccines. There are currently more than 300 trials exploring therapeutics for COVID-19, yet near universal exclusion of pregnant women, despite many of these trials repurposing drugs already widely, and safely, used in pregnancy. For example, hydroxychloroquine is commonly used in connective tissue disorders, and lopinavir plus ritonavir in combination is a common anti-retroviral therapy to prevent vertical transmission of HIV. Intrapartum azithromycin decreases maternal and neonatal infective morbidity. Interferon beta-1a reduces multiple sclerosis relapse. Exposure registry and post-marketing surveillance for these drugs all provide reassuring signals of safety.

To conclude; pregnant women should do whatever they can to avoid getting COVID-19 infection.

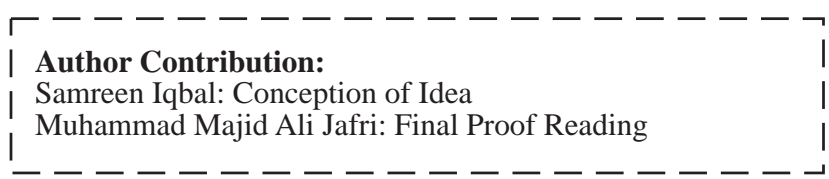

\section{REFERENCES:}

1. Breslin N, Baptiste C, Miller R, Fuchs K, Goffman D, et.al. Coronavirus disease 2019 in pregnancy: early lesson.American Journal of Obstetrics \& Gynecology MFM. 2020;2(2): supplement: 100111.

2. Whitehead CL, Walker SP. Consider pregnancy in COVID19 therapeutic drug and vaccine trials. The LANCET. 2020; 395(10237): E92.

3. Special considerations in Pregnancy and Post-Delivery. NIH COVID-19 Treatment Guidelines.
4. The American College of Obstetricians and Gynecologists. Outpatient assessment and management for pregnant women with suspected or confirmed novel coronavirus (COVID-19). 2020 .

5. Kristin S. Placentas from COVID-19-positive pregnant women show injury. Northwestern Now (II). 2020.

6. Poon LC, Yang H, Kapur A, Melamed N, Dao B, Divakar H et.al. Global interim guidance on coronavirus disease 2019 (COVID-19) during pregnancy and puerperium from FIGO and allied partners: Information form healthcare professionals. International Journal of Gynecology \& Obstetrics. 2020;149(3). https://doi.org/10.1002/ijgo. 13156.

7. Centers for Disease Control and Prevention. Interim considerations for infection prevention and control of coronavirus disease 2019 (COVID-19) in inpatient obstetric healthcare settings. 2020.

8. The American College of Obstetricians and Gynecologists. Practice advisory: novel coronavirus 2019 (COVID-19). 2020.

9. Society for Maternal Fetal Medicine. Coronavirus (COVID19) and pregnancy: what maternal fetal medicine subspecialists need to know. 2020.

10. Rasmussen SA, Smulian JC, Lednicky JA, Wen TS, Jamieson DJ. Coronavirus disease 2019 (COVID-19) and pregnancy: what obstetricians need to know. Am J Obstet Gynecol. 2020.

11. The American College of Obstetricians and Gynecologists. COVID-19 frequently asked questions for obstetriciansgynecologists, obstetrics. 2020.

12. Corona Virus infection and pregnancy. Information for pregnant women and their families.Royal College of Obstetricians \& Gynecologists.

13. Carvalho WBD, Gibelli MABC, Kerbs VLJ, Calil VMLT, Johnston C. Expert recommendations for the care of newborns of mothers with COVID-19. Clinics (Sao Paulo) 2020;75:e1932. doi: 10.6061/clinics/2020/e1932.

14. Wang SS, Zhou X, Lin XG, Liu YY, Wu, JL Sharifu LM et.al. Experience of Clinical Management for pregnant women and newborns with Novel Coronavirus Pneumonia in Tongji Hospital, China. Current Medical Science. 2020;40(2):2859. 